\title{
Terminal differentiation of human epidermal keratinocytes involves mitochondria- and caspase-dependent cell death pathway
}

\author{
Cell Death and Differentiation (2003) 10, 850-852. doi: 10.1038/s..cdd.4401245
}

\author{
Dear Editor,
}

The epidermis is the continuously renewing multilayered epithelium of the skin in which all steps of keratinocyte differentiation, from cell birth to cell death, can be identified by the cell position within the strata, its morphology and accompanying biochemical markers (for a review, see Fuchs ${ }^{1}$ and Polakowska and $\mathrm{Haake}^{2}$ ). Interestingly, some features of terminally differentiating keratinocytes resemble cellular changes associated with apoptosis. This implies that classical apoptosis is only one of several possible forms of cell death (see Leist and Jaattela ${ }^{3}$ ) and that keratinocyte terminal differentiation may be another. We attempted to establish whether keratinocyte differentiation involves components of the mitochondria- and caspase-dependent apoptotic machinery. An activation of the mitochondria-dependent cell death pathway is often manifested by a decrease in mitochondrial membrane potential. ${ }^{4}$ As shown in Figure $1 \mathrm{a}, \mathrm{Ca}^{2+}$ treatment of normal human keratinocytes (NHK) and HaCaT cells, an immortalized keratinocyte cell line still able to differentiate, resulted in a one log decrease in the red fluorescence of chloromethyl-X-rosamine (CMX-Ros) detected by flow cytometry analysis. This implies that differentiation of cultured epidermal keratinocytes correlates with a decrease in mitochondrial membrane potential $\left(\Delta \Psi_{\mathrm{m}}\right)$. Interestingly, the decrease was also observed in differentiating keratinocytes of epidermal cell suspension (ECS) freshly isolated from native human epidermis, although heterogeneity in the intensity of CMX-Ros fluorescence was apparent. In all cases, trypan blue and cell cycle analysis revealed that most cells were viable and only an insignificant fraction underwent cell death. Forward and side scatter analysis of viable ECS keratinocytes identified a smaller-cell sub-population, which consisted of cells with both lower and higher fluorescence intensity and a more homogeneous larger-cell subpopulation with low CMXRos fluorescence (Figure 1a, flow cytometry). Fluorescence microscopy (FM) analysis of ECS keratinocytes confirmed their heterogeneity in both size and fluorescence intensity (Figure 1a, FM). The heterogeneity of ECS keratinocytes suggested that they were probably a mixture of undifferentiated and differentiating cells. Their identity was determined by a comparative study of the CMX-Ros staining with markers of keratinocyte differentiation (not shown). This analysis established a clear correlation between the intensity of CMX-Ros fluorescence and keratinocyte phenotype. The small, bright cells were mostly undifferentiated basal keratinocytes (Figure 1a, FM-cells 1 and 2), whereas the larger cells, fluorescing less intensely, were identified as keratino- cytes undergoing terminal differentiation (Figure 1a, FM-cells 3 and 4). The size of the differentiating keratinocytes varied and correlated inversely with the net accumulation of the CMX-Ros dye, supporting the in vitro findings. More subtle changes in the subcellular distribution and morphology pattern of mitochondria could also be observed, ranging from the punctiform individual structures dispersed throughout the cytoplasm in a majority of cells to fine points at the cell periphery and around the nucleus of large flat cells (Figure 1a, FM). Most mitochondria clustered near the nucleus appeared dysfunctional as revealed by a decrease in CMX-Ros staining, whereas some remained polarized at all times, even in the dim and flat terminally differentiated cells as judged by a bright punctuated CMX-Ros staining in the cell periphery. Mitochondria were identified with MitoTracker Green, which, contrary to CMX-Ros, is not sensitive to the mitochondria membrane potential and is a morphological - as opposed to functional dye. This preliminary finding and observations made by others in differentiating osteoblasts and lens (see Komarova et a $\bar{P}$ and Sanders and Parker $^{6}$ ) as well as in apoptotic cells, ${ }^{7}$ support the concept of mitochondrial heterogeneity within the cell. According to the literature, a differential response of mitochondria to environmental signals may maintain a functional electron transport chain in a subset of mitochondria to produce the energy required for the degradative processes during late apoptosis, ${ }^{7}$ terminal differentiation of lens ${ }^{6}$ and perhaps also that of keratinocytes. The above data suggest that mitochondrial membrane potential, mitochondrial phenotype and subcellular distribution are modified during keratinocyte differentiation.

Interestingly, the mitochondrial changes in aspect of subcellular distribution observed during keratinocyte differentiation appear similar to those reported during classical apoptosis. ${ }^{4}$ Also, in analogy to classical apoptosis events, $\Delta \Psi_{\mathrm{m}}$, in differentiated keratinocytes may indicate abnormally functioning mitochondria often associated with cytochrome $c$ release from the mitochondrial intermembrane space to the cytoplasm. $^{3,4}$ However, unlike in apoptosis where all mitochondria release cytochrome $c{ }^{8}$ we observed a gradual release of cytochrome $c$ during epidermal maturation. Thus, in ECS keratinocytes, a cytochrome $c$ redistribution correlated with cellular size and the expression of differentiation markers: it was punctuated in small cells (Figure 1b, cell 1) and punctuated or diffuse in larger cells (Figure 1b, cell 2). A diffuse staining with anticytochrome $c$ antibody was detected mainly in large flat cells (Figure 1b, cell 3), corresponding to 
a

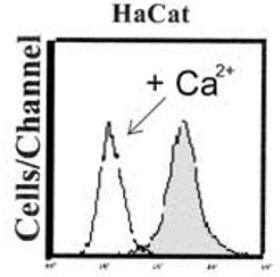

NHK

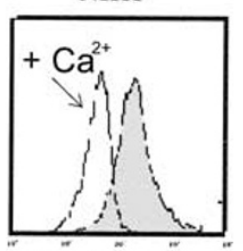

CMX Ros
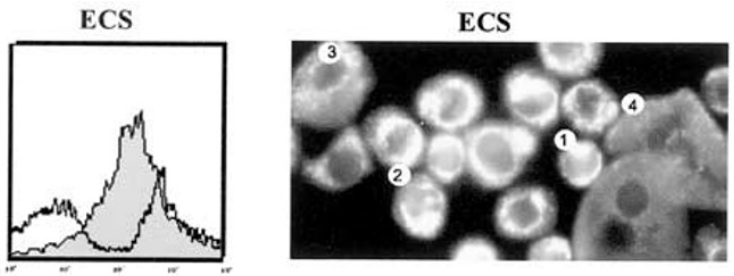

b
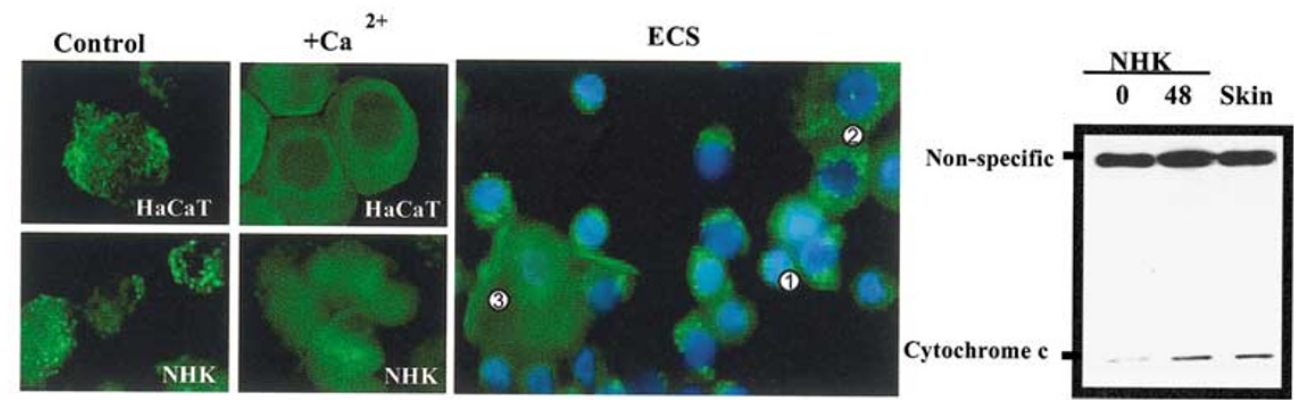

C
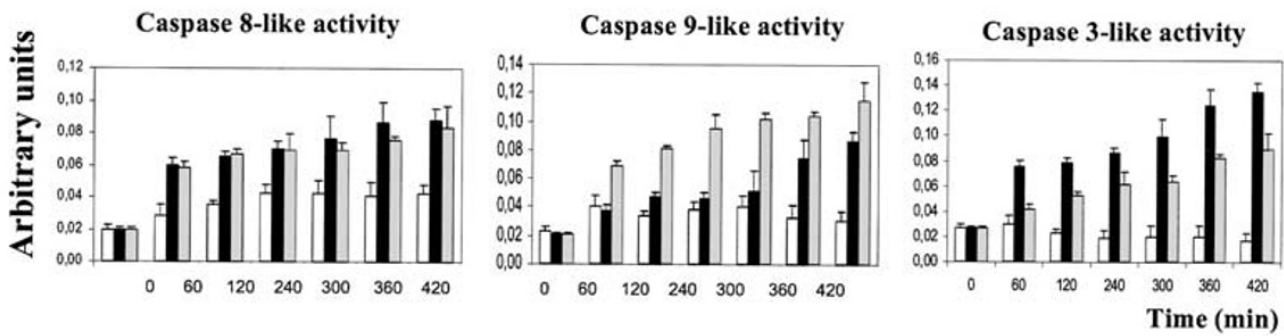

d
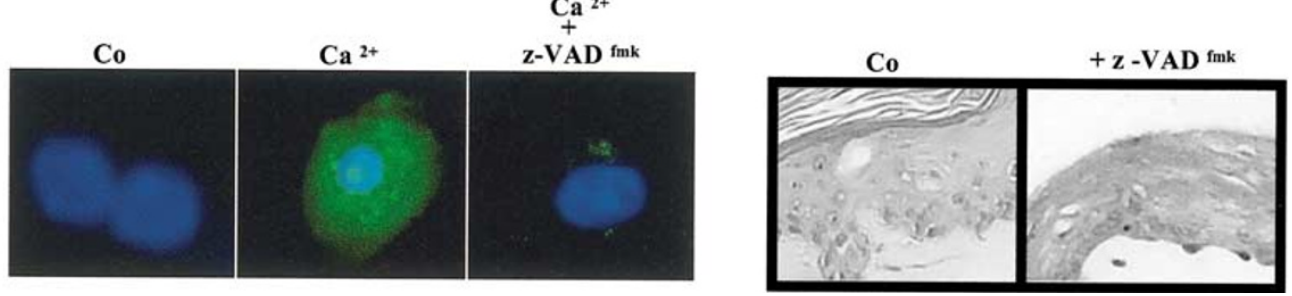

Figure 1 (a) Primary normal human keratinocytes (NHK) obtained from adult skin as described before ${ }^{13}$ and HaCaT cell line were incubated either in SFM medium (gray profile) or SFM+1.2 $\mathrm{mM} \mathrm{CaCl}_{2}$ (white profile). HaCaT, $\mathrm{Ca}^{2+}$-treated NHK and undifferentiated (gray profile) and differentiating (white profile) keratinocytes of freshly isolated epidermal cell suspension (ECS) were labeled with the potential sensitive $50 \mathrm{nM}$ cationic CMX-Ros and immediately analyzed by flow cytometry or fluorescent microscopy (FM). Control experiments were performed in the presence of $50 \mu \mathrm{M}$ carbamoycyanide $m$-chloro phenyl hydrazone $(\mathrm{mClCCP})$, an uncoupling agent that abolishes the mitochondrial membrane potential $\left(\Delta \Psi_{\mathrm{m}}\right)$. To obtain cell populations enriched in undifferentiated and differentiated keratinocytes, ECS was plated on collagen IV-coated plates and cells were allowed to adhere for $2 \mathrm{~h}$. Then unattached (differentiating, FM panel, cells 3 and 4 ) cells were collected directly from the supernatant by centrifugation while the attached (undifferentiated, FM panel, cells 1 and 2) keratinocytes were harvested by trypsinization. (b) Untreated cells show punctuated staining with cytochrome $c$ antibody (Pharmingen, clone 6H2.B4), while some of the calcium-treated cells show diffuse staining of cytochrome $c$. A transition from the punctuated (cells 1 and 2) to the diffuse (cell 3) staining is also seen in the ECS. Western blot was using anti-cytochrome $c$ antibody (Pharmingen, clone $7 \mathrm{H} 8.2 \mathrm{C} 12$ ) and S-100 cell fraction of cytosols from the NHK induced to differentiate by $1.2 \mathrm{mM} \mathrm{Ca}^{2+}$ for $48 \mathrm{~h}$ and from a whole epidermis (skin). (c) A colorimetric assay of Ac-DEVD-pNA (caspase-3-like activity), Ac-IETD-pNA (caspase-8-like activity) and Ac-LEHD-pNA (caspase-9-like activity) substrates (Biomol, Plymouth, PA, USA) was performed for $420 \mathrm{~min}$ using $100 \mu \mathrm{g}$ protein lysates from the epidermis (black bars) and $\mathrm{Ca}^{2+}$-induced (gray bars) and undifferentiated NHK (white bars). A negative control was performed without any lysate. (d) The assay detecting active caspases in situ was performed using a fluorescent substrate of activated caspases, the CaspACE ${ }^{\mathrm{TM}}$ FITC-VAD ${ }^{\mathrm{fmk}}$ (Promega). The substrate was added to NHK grown in the presence and absence of $1.2 \mathrm{mM} \mathrm{Ca}^{2+}$ and $z-V^{\mathrm{f}} \mathrm{D}^{\mathrm{fmk}}$ for $48 \mathrm{~h}$ at a final concentration of $5 \mu \mathrm{M}$. After incubation, the cells were stained with Hoechst $33342(1 \mu \mathrm{g} / \mathrm{ml}$, Molecular Probes) to visualize nuclei and immediately analyzed under a fluorescence microscope (left panel). Addition of $100 \mu \mathrm{M} \mathrm{z-VAD^{ \textrm {fmk } }}$ at the time of rafting inhibited maturation of the skin equivalent (SE) epidermis prepared as described previously. ${ }^{13}$ Sections of paraffin-embedded skin equivalents were stained with hemaotoxylin and eosin (right panel)

the upper epidermis. Immunohistochemistry of paraffinembedded epidermis revealed that cytochrome $c$ staining switched gradually from punctuated to punctuated and diffuse and to mostly diffuse as keratinocytes differentiated (not shown). This suggests that cytochrome $c$ could be released selectively from some mitochondria and that its limited release could have already occurred in suprabasal layers. The selective and progressive cytochrome $c$ release during 
keratinocyte differentiation is consistent with the concept of mitochondrial heterogeneity, and proves particularly interesting because it may correlate with a vector of the calcium gradient in the epidermis. In cultured, undifferentiated keratinocytes, FITC-cytochrome $c$ staining was cytoplasmic and predominantly punctuated, whereas in some $\mathrm{Ca}^{2+}$ induced keratinocytes, this staining became uniformly diffused (Figure 1b). Western blot analysis confirmed that the cytosol prepared from differentiating NHK or from whole epidermis contained free cytochrome $c$ (Figure $1 b$ ), supporting a possible connection between cytochrome $c$ release and keratinocyte differentiaton. Cytochrome $c$ release, however, did not seem to coincide with the immediate activation of caspases or with cell death, as it does in classical mitochondrial-dependent apoptosis. For in keratinocytes, activation of caspases and cell death do not occur until the cells reach the stage of terminal differentiation. It is important to note that cytochrome $c$ release without immediate cell death has been reported in other cell types (see Von Ashen et $a l^{8}$ ), where it was associated with the cellular differentiation process. $^{9}$

Although it is difficult to say precisely when cytochrome $c$ is released at a significant level, and how long keratinocytes can stand cytochrome $c$ in their cytoplasm without entering the final death process, the topographic organization of the differentiation pathway within the epidermal strata provides a unique opportunity to determine the sequence and relative time of induction of apoptotic markers during epidermal maturation. We demonstrated by means of various techniques that cleaved caspase- 3 is present in terminally differentiating keratinocytes and may contribute to the degradation process in the late stages of epidermal cell differentiation. This concords with the earlier findings of Weil et al. ${ }^{10}$ Active caspases were present in $\mathrm{Ca}^{2+}$-induced keratinocytes but not in undifferentiated cells as determined using fluorescent substrate FITC-VAD ${ }^{\text {fink }}$ (Figure 1d, left). Their activity was inhibited by the general caspase inhibitor benzyloxycarboylVal-Ala-Asp-fluoromethylketone (z-VAD $\left.{ }^{\mathrm{ftnk}}\right)$, confirming caspase specificity of the fluorescent substrate. To identify the active caspases, a colorimetric assay with Ac-DEVD-pNA, Ac-IETD-pNA and Ac-LEHD-pNA as substrates for caspase$3,-8$ and -9 , respectively, was performed using lysates from undifferentiated and differentiating keratinocytes. Data in Figure 1c demonstrate that lysates from differentiating - but not from undifferentiated - keratinocytes displayed caspase activity. Caspase-3 activation was also investigated by Western blot analysis (not shown). The cleaved active form of caspase-3 was clearly present in the selected differentiating keratinocytes of ECS and at a low level in the entire epidermis, implying that this enzyme was activated only in a small subpopulation of the epidermal cells. An immunohistochemistry analysis (not shown) of the human epidermis detected the active caspase- 3 antigen in one layer of the upper stratum granulosum, where flat keratinocytes undergo the degradation process. Our preliminary evidence and findings in several other cell systems (see Zeuner et all ${ }^{11}$ ) show that caspases may regulate cell growth and differentiation in addition to their well-known proapoptotic and proin- flammatory function, and suggest that perhaps in continuously renewing tissues, the apoptotic machinery is recruited to the differentiation program converting live cells into dead, terminally differentiated functional units. Very importantly, our functional study using an in vivo-like skin equivalent model demonstrated that $z V A D^{\mathrm{fmk}}$ present at the time of rafting, the stimulatory condition for keratinocyte stratification and terminal differentiation, prevented the formation of normal stratum corneum (Figure 1d, right), suggesting that indeed the degradation stage may be caspase-dependent, although caspase-independent processes cannot be excluded.

We conclude that the differentiation process in epidermal keratinocytes probably utilizes mitochondrial-dependent cell death machinery as it proceeds. The spatial-temporal coordination of the differentiation program with the apoptotic machinery implies that these two processes are tightly linked. It can be speculated that accommodation of the apoptotic machinery to the differentiation program requires that differentiating keratinocytes be equipped with inhibitors of the apoptotic pathway to temporarily prevent cell death and allow for the expression of the differentiated phenotype. It has recently been reported that differentiating keratinocytes express several antiapoptotic factors such as heat shock proteins (HSP) 27 and 70 or inhibitors of apoptosis (IAP). ${ }^{12}$ We further speculate that since differentiation-induced cell death in keratinocytes involves the apoptotic machinery, this machinery has to be negatively controlled and de-repressed stepwise during epidermal maturation so that a particular step of the mitochondrial-dependent cell death pathway corresponds to the specific stage of keratinocyte differentiation. More generally, our concept is that differentiation is regulated by a subtle balance of repression and activation between proliferation and apoptosis.

\section{Allombert-Blaise ${ }^{1,2}$, S Tamiji ${ }^{1}$, L Mortier $^{1,2}$, H Fauvel $^{1}$, M Tual' ${ }^{1}$, E Delaporte $^{2}$, F Piette $^{2}$, E Martin DeLassale ${ }^{3}$, $P$ Formstecher ${ }^{1}, P$ Marchetti $^{1}$ and $R$ Polakowska ${ }^{*, 1}$ \\ ${ }^{1}$ INSERM U459, Faculté de Médecine 1, Lille Cedex, France \\ 2 Clinique de Dermatologie, Hôpital Huriez, CHRU, 59037 Lille Cedex, France \\ ${ }^{3}$ Service d'Anatomie et Cytologie Pathologiques, Hopital Calmette, Lille Cedex, France \\ * Corresponding author: R Polakowska, INSERM U459, Faculté de Médecine 1, Place de Verdun, 59045 Lille Cedex, France}

1. Fuchs E (1990) J. Cell Biol. 111: 2807-2814.

2. Polakowska R and Haake AR (1994) Cell Death Differ. 1: 19-31.

3. Leist M and Jaattela M (2001) Nat. Rev. Mol. Cell Biol. 2: 589-598.

4. Desagher S and Martinou JC (2000) Trends Cell. Biol. 10: 369-377.

5. Komarova SV et al. (2000) Am. J. Physiol. Cell Physiol. 279: 1C12201 C1229.

6. Sanders EJ and Parker E (2002) J. Anat. 201: 121-135.

7. Halestrap AP et al. (2002) Biochimie 84: 153-166.

8. Von Ashen $O$ et al. (2000) Cell Death Differ. 7: 1192-1199.

9. Yu XH et al. (2002) Cell Death Differ. 9: 189-198.

10. Weil M et al. (1999) Curr. Biol. 9: 361-364.

11. Zeuner A et al. (1999) Cell Death Differ. 6: 1075-1080.

12. Jansen BJ et al. (2001) J. Invest. Dermatol. 116: 12-22.

13. Haake A and Polakowska R (1995) Cell Death Differ. 2: 183-193. 